\title{
Overexpression of astrocyte-elevated gene- 1 is associated with ovarian cancer development and progression
}

\author{
BO ZHOU ${ }^{1,2^{*}}$, JUE YANG $^{1 *}$, BIN SHU $^{2}$, KUNMEI LIU $^{3}$, LEZHEN XUE $^{2}$, NING SU $^{2,4}$, JING LIU $^{2}$ and TAO XI ${ }^{1}$ \\ ${ }^{1}$ State Key Laboratory of Natural Medicines, School of Life Science and Technology, China Pharmaceutical University; \\ ${ }^{2}$ Department of Toxicology, Jiangsu Center of Safety Evaluation for Drugs, School of Pharmaceutical Sciences, \\ Nanjing University of Technology, Nanjing, Jiangsu 210009; ${ }^{3}$ Ningxia Key Laboratory of Cerebrocranial Diseases, \\ School of Laboratory Medicine, Ningxia Medical University, Yinchuan, Ningxia 750004; ${ }^{4}$ Department of Pathology, \\ Zhongda Hospital, Southeast University, Nanjing, Jiangsu 210009, P.R. China
}

Received February 3, 2014; Accepted November 7, 2014

DOI: $10.3892 / \mathrm{mmr} .2014 .3056$

\begin{abstract}
It has previously been reported that astrocyte-elevated gene-1 (AEG-1) has a critical role in the regulation of tumor development, and/or progression. However, the functional significance of AEG-1 in human ovarian cancer remains unclear. The present study conducted an immunohistochemical analysis of ovarian tissues, and the association between AEG-1 protein expression, clinicopathological features and outcomes were investigated. The gain or loss of AEG-1 function was also examined, through exogenous overexpression or knockdown of expression by small interfering RNA, in ovarian cancer cells. Normal ovarian tissue exhibited very little or no AEG-1 immunoreactivity, whereas high expression levels of AEG-1 were detected in $12.7 \%$ of cystadenomas, $30.0 \%$ of borderline tumors, and $71.2 \%$ of ovarian carcinomas, respectively, as determined by immunohistochemistry. Statistical analyses demonstrated a significant correlation of AEG-1 expression with differentiation $(\mathrm{P}=0.001)$, lymph node metastasis $(\mathrm{P}=0.008)$ and clinical staging $(\mathrm{P}=0.002)$. In addition, the overall survival time of patients with higher AEG-1 expression levels was markedly shorter, as compared with patients with lower expression levels of AEG-1 $(\mathrm{P}=0.001)$. Multivariate analysis indicated
\end{abstract}

Correspondence to: Professor Tao Xi, State Key Laboratory of Natural Medicines, School of Life Science and Technology, China Pharmaceutical University, 24 Tong Jia Xiang, Nanjing, Jiangsu 210009, P.R. China

E-mail: newgansal@hotmail.com

Dr Jing Liu, Department of Toxicology, Jiangsu Center of Safety Evaluation for Drugs, School of Pharmaceutical Sciences, Nanjing University of Technology, 24 Ma Jia Jie, Nanjing, Jiangsu 210009, P.R. China

E-mail: jansens@foxmail.com

*Contributed equally

Key words: astrocyte-elevated gene-1, ovarian cancer, proliferation, metastasis that AEG-1 expression was an independent prognostic indicator of the survival of patients with ovarian cancer. Furthermore, exogenous overexpression of AEG-1 in ovarian cancer cells was shown to significantly enhance cell proliferation, adhesion and invasion. Conversely, silencing AEG-1 expression caused an inhibition of cell growth, adhesion and invasion. The results of the present study indicate that AEG-1 is a valuable biomarker for the prediction of ovarian cancer prognosis, and AEG-1 inhibition may be a potential therapeutic strategy for ovarian cancer treatment.

\section{Introduction}

Ovarian cancer is the most lethal gynecological malignancy worldwide. According to the International Agency for Research on Cancer for 2008, 225,500 individuals were diagnosed with ovarian cancer and 140,200 cases were fatal (1). Epithelial ovarian cancer accounts for $\sim 90 \%$ of all ovarian malignancies, and the majority of patients are asymptomatic until the later stages of the disease, contributing to the high levels of mortality associated with this disease $(2,3)$. The precise factors that initiate ovarian carcinogenesis remain unclear, however it is generally accepted that ovarian cancer has numerous etiological factors, including genetic alteration, hormonal factors and some life-style factors $(4,5)$.

Despite rapid advancement of medical and surgical treatments, as well as the development of novel drugs and chemotherapy regimens for patients with ovarian cancer, the prognosis has not significantly improved. The average five-year survival rate of patients with advanced stage ovarian cancer ranges between 20 and 30\% (6). The pathogenesis of ovarian carcinoma is a multi-step process, including oncogene activation and/or inactivation of tumor suppressor genes (7). Ovarian cancer has been widely studied; however, the search for specific gene alterations has been insufficient and the identification of molecular markers that are present in ovarian carcinoma cells, which may serve as reliable biomarkers, remains limited.

Astrocyte-elevated gene (AEG)-1, also known as metadherin and LYRIC/3D3, was originally cloned and characterized as a human immunodeficiency virus-1-inducible gene, in 
primary human fetal astrocytes $(8,9)$. Elevated expression of AEG-1 has been observed in various types of human cancer, including breast, esophageal, hepatocellular, colorectal, prostate and ovarian cancer (10-15). Furthermore, previous studies have shown that AEG-1 has an important role in cell growth and proliferation, angiogenesis, chemoresistance, invasion, and metastasis by activating numerous signaling pathways (16-18), such as phosphoinositide 3-kinase (PI3K)-Akt $(11,14)$ nuclear factor-кB (19), and mitogen activated protein kinase (MAPK) and Wnt pathways (12). Notably, AEG-1 itself is a downstream target molecule of oncogenic Ha-ras and c-myc, and mediates their growth promoting effects (20). However, studies regarding the underlying mechanisms of AEG-1 in ovarian cancer are lacking.

In the present study, immunohistochemistry (IHC) was used to investigate the expression of AEG-1 in patients with ovarian cancer and its association with certain clinical parameters. In addition, the effect of AEG-1 on ovarian cancer cells was also evaluated.

\section{Materials and methods}

Cell lines and culture conditions. SKOV-3 human ovarian cancer cells from the American Type Culture Collection (Manassas, VA, USA) were cultured in RPMI-1640 medium (Gibco Life Technologies, Carlsbad, CA, USA) supplemented with $10 \%$ fetal bovine serum (FBS; Gibco Life Technologies Carlsbad, CA, USA), $100 \mathrm{U} / \mathrm{ml}$ penicillin (KeyGen, Nanjing, China), and $100 \mathrm{mg} / \mathrm{l}$ streptomycin (KeyGen), in a humidified atmosphere containing $5 \% \mathrm{CO}_{2}$ at $37^{\circ} \mathrm{C}$.

Patients and tissues. Formalin-fixed and paraffin-embedded tissue samples from 138 patients with epithelial ovarian tumors were obtained from the archives of the Department of Pathology, Zhongda Hospital, Southeast University (Nanjing, China). The tumor samples included epithelial ovarian cancer $(n=73)$, borderline tumor $(n=10)$ and benign cystadenoma $(\mathrm{n}=55)$. In addition, 10 normal ovaries from hysterectomy specimens were resected for non-ovarian disease, in Zhongda Hospital (Nanjiing, China), and were analyzed by IHC. None of the patients had received chemotherapy prior to surgery.

The age of the patients with ovarian cancer ranged between 38 and 82 years, with a median age of 56 years. Tumor staging was conducted according to the International Federation of Gynecology and Obstetrics (FIGO) system (21). Tumors were assessed according to the Silverberg grading system. The clinicopathological features of the patients with ovarian cancer, including age, histological type, differentiation degree, lymph node metastasis and clinical stage are summarized in Table I. The study was approved by the Ethics Committee of Zhongda Hospital Affiliated to Southeast University, and conducted in accordance with the principles defined in the Declaration of Helsinki. Informed consent was exempted by the board due to the retrospective nature of the research. All information regarding the human material used in the present study was managed using anonymous numerical codes.

Antibodies. Rabbit monoclonal anti-AEG-1 antibody was obtained from Epitomics (dilution 1:1,000; Burlingame, CA, USA). Rabbit polyclonal anti-AKT, anti-phospho-AKT
(Ser473), anti-extracellular signal-regulated kinase (ERK1/2), anti-phospho-ERK1/2 (Tyr204), anti-glycogen synthase kinase (GSK)/3 $\beta$, anti-phospho-GSK3 $\beta$ (Ser9), anti-forkhead box (FOXO) $3 \alpha$ and anti-phospho-FOXO3 $\alpha$ (Ser253) antibodies were obtained from Signalway Antibody (College Park, Maryland, USA). All were diluted to 1:1,000. Rabbit polyclonal anti- $\beta$-actin was purchased from ZSGB-Bio (Beijing, China).

Small interfering (si)RNA transfection. A pcDNA3.1 vector overexpressing human AEG-1 was generated by subcloning the PCR-amplified human AEG-1 coding sequence into the pcDNA3.1 vector (Ambion, Austin, TX, USA). To silence endogenous AEG-1, the AEG-1 siRNA oligonucleotides (sense 5'-GUUACCACCGAGCAACUU ADTDT-3' and antisense 5'-UAAGUUGCUCGGUGGUAA CDTDT-3') were synthesized by Biomics Biotechnologies (Biomic, Nantong, China). The control cells were transfected with universal negative control (NC) siRNA (Biomics, Jiang Su, China). The siRNA transfection of the SKOV3 cells was performed in six-well plates using Lipofectamine ${ }^{\circledR}$ 2000 (Invitrogen Life Technologies, Invitrogen, CA, USA), according to the manufacturer's instructions.

Immunohistochemical staining. Immunohistochemical staining was performed to study protein expression levels in all of the tissue samples, using the Ultrasensitive S-P kit and diaminobenzidine (Maixin-Bio Co., Fuzhou, China). Briefly, unstained $4 \mu$ m tissue sections were cut from the selected paraffin blocks and deparaffinized by routine techniques (22). Antigen retrieval was performed by placing the slides in boiling citric acid buffer (KeyGen) at pH 6.0 for $10 \mathrm{~min}$, the slides were then treated with 3\% hydrogen peroxide (Maixin-Bio Co., Fuzhou, China) to quench the endogenous peroxidase activity, followed by incubation with $10 \%$ normal goat serum (Maixin-Bio Co.) to block the non-specific binding. The slides were then incubated with a primary antibody specifically targeting AEG-1 (dilution 1:200; Epitomics, Burlingame, CA, USA) overnight at $4^{\circ} \mathrm{C}$. The sections were then washed with PBS and incubated with a biotin-labeled secondary antibody (Maixin-Bio Co.), followed by horseradish peroxidase-conjugated streptavidin (Maixin-Bio Co.) for $10 \mathrm{~min}$. The slides were then exposed to a 3,3'-diaminobenzidine tetrahydrochloride substrate kit (ZSGB-Bio) and counterstained with hematoxylin (KeyGen).

Staining assessment and scoring. The AEG-1 expression levels were classified semi-quantitatively, based on the proportion of positively stained tumor cells and the intensity of the staining. The proportion of positive cells was scored as follows: 0 , no positive tumor cells; $1,<10 \%$ positive tumor cells; $2,10-50 \%$ positive tumor cells; and $3,>50 \%$ positive tumor cells. The intensity of staining was graded according to the following criteria: 0 , no staining; 1 , weak staining; 2 , moderate staining; and 3, strong staining. The staining intensity score was multiplied by the percentage of positive tumor cells to calculate the protein expression levels. A score of $\geq 4$ was considered to indicate high AEG-1 expression, whereas a score of $\leq 3$ was considered to indicate low expression. The stained sections were scored in duplicate by two independent investigators who were blinded to the histopathological features, and patient data of the samples. 
Table I. Results of immunohistochemistry and clinicopathological characteristics of the patients.

\begin{tabular}{|c|c|c|c|c|}
\hline \multirow[b]{2}{*}{ Characteristic } & \multirow[b]{2}{*}{ Cases (n) } & \multicolumn{2}{|c|}{ Astrocyte-elevated gene-1 } & \multirow[b]{2}{*}{ P-value } \\
\hline & & Low expression no. $(\%)$ & High expression no. $(\%)$ & \\
\hline Total & & & & $<0.001$ \\
\hline Benign cystadenomas & 55 & 48 & 7 & \\
\hline Borderline tumors & 10 & 7 & 3 & \\
\hline Carcinomas & 73 & 21 & 52 & \\
\hline Age (years) & & & & 0.193 \\
\hline$\leq 55$ & 33 & 12 & 21 & \\
\hline$>55$ & 40 & 9 & 31 & \\
\hline Histological type & & & & 0.214 \\
\hline Serous & 55 & 13 & 42 & \\
\hline Mucinous & 8 & 4 & 4 & \\
\hline Other & 10 & 4 & 6 & \\
\hline Differentiation degree (Silveberg) & & & & 0.004 \\
\hline G1 & 7 & 5 & 2 & \\
\hline $\mathrm{G} 2$ & 30 & 11 & 19 & \\
\hline G3 & 36 & 5 & 31 & \\
\hline Lymph node metastasis & & & & 0.009 \\
\hline No & 54 & 20 & 34 & \\
\hline Yes & 19 & 1 & 18 & \\
\hline Clinical stage & & & & 0.006 \\
\hline I & 17 & 10 & 7 & \\
\hline II & 15 & 4 & 11 & \\
\hline III/IV & 41 & 7 & 34 & \\
\hline
\end{tabular}

Other, endometrioid adenocarcinoma and clear-cell carcinoma; FIGO indicates international federation of gynecology and obstetrics.

Western blotting. Total cellular proteins were extracted using lysis buffer [50 mmol/l Tris, $\mathrm{pH} 7.4,150 \mathrm{mmol} / \mathrm{l} \mathrm{NaCl}$, $1 \%$ Triton $\mathrm{X}-100,1 \%$ deoxycholic phenylmethylsulfonyl fluoride, $1 \mathrm{mg} / \mathrm{ml}$ of aprotinin, $5.0 \mathrm{~mm}$ sodium pyrophosphate, $1.0 \mathrm{~g} / \mathrm{ml}$ leupeptin, $0.1 \mathrm{~mm}$ phenylmethylsulfonyl fluoride, and $1 \mathrm{~mm} / \mathrm{l}$ of DTT (KeyGen)]. The protein samples $(40 \mu \mathrm{g})$ were then separated by $10 \%$ SDS-PAGE and electrophoretically transferred onto polyvinylidene difluoride membranes (Beyotime Institute of Biotechnology, Haimen, China). The membranes were then incubated with the primary antibodies: Anti-AEG-1, anti-AKT, anti-phospho-AKT (Ser473), anti-ERK1/2, anti-phospho-ERK1/2 (Tyr204), anti-GSK3 $\beta$, anti-phospho-GSK3 $\beta$ (Ser9), anti-FOXO3a and antiphospho-FOXO3a (Ser253), in 5\% milk/Tris-buffered saline-Tween ${ }^{\circledR} 20$ (TBST) for $24 \mathrm{~h}$ at $4^{\circ} \mathrm{C}$. Following washing with TBST, the membranes were incubated with secondary antibody for $1 \mathrm{~h}$ at room temperature. The bands were visualized using enhanced chemiluminescence detection reagents (GE Healthcare Life Sciences, Uppsala, Sweden). Protein levels were quantified by density analysis using Quantity One software version 4.6 (Bio-Rad Laboratories, Inc., Hercules, CA, USA).

$R N A$ extraction and reverse transcription quantitative polymerase chain reaction ( $R T-q P C R)$. Total RNA was extracted from the cultured cells using TRIzol ${ }^{\circledR}$ reagent (Invitrogen Life Technologies), according to the manufacturer's instructions. A total of $1 \mu \mathrm{g}$ RNA from each sample was reverse transcribed into cDNA using random hexamers (Beyotime Institute of Biotechnology). PCR amplification was carried out in a total volume of $25 \mu \mathrm{l}$, containing $0.5 \mu \mathrm{l}$ each primer, $12 \mathrm{ml}$ SYBR $^{\circledR}$ Green qPCR Master Mix (Takara Biotechnology, Dalian, China) and $2 \mu 1$ 1:12.5 diluted cDNA. Generation of standard curves and qPCR were carried out using an ABI7500 Real-Time PCR instrument (Applied Biosystems Life Technologies, Foster City, CA, USA). The sequences of the primers used were as follows: AEG-1, forward 5'-AAATAG CCAGCCTATCAAGACTC-3', reverse 5'-TTCAGACTTGGT CTGTGAAGGAG-3'; and GAPDH, forward 5'-AAGGTC GGAGTCACCGGATT-3', and reverse 5'-CTGGAAGATGGT GATGGGATT-3'. Expression data were normalized to the geometric mean of the housekeeping gene GAPDH, in order to control the variability in expression levels. The cycle threshold $(\mathrm{Ct})$ values were measured and gene expression levels were analyzed using the $2^{-\Delta \Delta C T}$ method (23).

Cell proliferation assay. Cell growth was analyzed using the Cell Counting kit-8 (Beyotime Institute of Biotechnology, Haimen, China), according to the manufacturer's instructions. 
Table II. Spearman correlation analysis between astrocyte elevated gene-1 (AEG-1) and clinicopathological factors.

AEG-1 expression

Variable

Spearman correlation $(\mathrm{P})$

Age (years)

$0.150(0.207)$

Histological type

$-0.189(0.110)$

Differentiation degree

$0.370(0.001)$

Lymph node metastasis

$0.308(0.008)$

Clinical stage

Briefly, 2.0x10 3 cells were plated in each well of a 96-well plate. The cells were incubated at $37^{\circ} \mathrm{C}$ in an atmosphere containing $5 \% \mathrm{CO}_{2}$. The media in each well was then substituted with $100 \mathrm{ml}$ fresh medium, containing 10\% Cell Counting kit-8, and the cultures were incubated at $37^{\circ} \mathrm{C}$ for $1 \mathrm{~h}$. The absorbance value was determined using an automatic plate reader (Molecular Devices, Sunnyvale, CA, USA) at a wavelength of $450 \mathrm{~nm}$. All assays were carried out in triplicate.

Flow cytometry analysis. Detection of apoptosis and cell cycle distribution by flow cytometry was performed using the Cell Cycle and Apoptosis Analysis kit (Beyotime Institute of Biotechnology). The transfected cells were harvested by trypsinization and fixed with cold $70 \%$ ethanol at $4^{\circ} \mathrm{C}$ for $24 \mathrm{~h}$. Staining was performed according to the manufacturer's instructions. Flow cytometry (BD Biosciences, San Jose, CA, USA) was performed immediately after staining.

Adhesion assay. Microtiter wells were coated with Matrigel ${ }^{\mathrm{TM}}$ Basement Membrane Matrix (BD Biosciences) at $37^{\circ} \mathrm{C}$ for $4 \mathrm{~h}$, and then blocked for $1 \mathrm{~h}$ at $37^{\circ} \mathrm{C}$ with $0.5 \%$ bovine serum albumin in phosphate-buffered saline. The cells were seeded in triplicate at a density of $4 \times 10^{4}$ cells/well. The adherent cells were stained and examined using Cell Counting kit-8. The absorbance value was determined using an automatic plate reader (Molecular Devices) at $450 \mathrm{~nm}$.

Transwell $^{\circledR}$ invasion assay. The Transwell ${ }^{\circledR}$ migration assay was carried out using 24-well BioCoat ${ }^{\mathrm{TM}}$ cell culture inserts with $8 \mu \mathrm{m}$ pores (BD Biosciences) coated with a 1:5 dilution of Matrigel ${ }^{\mathrm{TM}}$. Briefly, the Matrigel ${ }^{\mathrm{TM}}$ was allowed to rehydrate for $2 \mathrm{~h}$ at $37^{\circ} \mathrm{C}$. The cells $\left(1 \times 10^{5}\right)$ were suspended in $200 \mu \mathrm{l}$ RPMI-1640 (1\% FBS) and seeded in triplicate into the upper chamber, and $800 \mu 1$ RPMI-1640 (10\% FBS) was added to the lower chamber. The invasion assay was performed at $37^{\circ} \mathrm{C}$ in a $5 \% \mathrm{CO}_{2}$ humidified incubator for $24 \mathrm{~h}$. Following the $24 \mathrm{~h}$ incubation, the cells on the upper surface of the membrane were wiped off, whereas the cells on the lower side were fixed with $4 \%$ paraformaldehyde and stained with $0.1 \%$ crystal violet (Beyotime Institute of Biotechnology). The number of cells was counted in five random fields per well, under a light microscope (Olympus Corporation, Tokyo, Japan).

Statistical analysis. All statistical analyses were carried out using SPSS version 17.0 statistical software package (SPSS,

Inc., Chicago, IL, USA). The $\chi^{2}$ test was used to analyze the association between AEG-1 expression levels and clinicopathological characteristics of the patients. Bivariate correlations between variables were calculated by Spearman's correlation coefficients. Differences in patient survival were determined using the Kaplan-Meier method and the log-rank test. Cox regression analysis (proportional hazard model) was used for the multivariate analysis of independent prognostic factors. $\mathrm{P}<0.05$ was considered to indicate a statistically significant difference.

\section{Results}

AEG-1 expression in ovarian tissues. Immunoreactivity for AEG-1 was examined primarily in the cytoplasm of ovarian surface epithelial and tumor cells. The normal ovarian tissue exhibited very little or no AEG-1 immunoreactivity (Fig. 1A). Whereas, high expression of AEG-1 was detected in benign cystadenomas (12.7\%), borderline tumors (30.0\%), and ovarian carcinomas $(71.2 \%)(\mathrm{P}<0.001$, Table I, Fig. 1B-D). AEG-1 expression was significantly associated with the degree of differentiation $(\mathrm{P}=0.004)$, lymph node metastasis $(\mathrm{P}=0.009)$ and clinical staging $(\mathrm{P}=0.006)$, however, it was not associated with age $(\mathrm{P}=0.193)$ or histological type $(\mathrm{P}=0.214)$. Spearman correlation analysis was further performed to confirm the correlation between AEG-1 expression and clinicopathological features. As shown in Table II, Spearman correlations of AEG-1 expression levels to degree of differentiation, lymph node metastasis and clinical staging were $0.370(\mathrm{P}=0.001)$, $0.308(\mathrm{P}=0.008)$ and $0.349(\mathrm{P}=0.002)$, respectively. These results suggest that high expression levels of AEG-1 may be closely associated with the clinical progression of ovarian cancer.

Association between AEG-1 expression and prognosis. Follow-up information was available on all 73 patients with ovarian carcinoma for periods ranging between 5 and 80 months (average=40.6). Survival curves for patients with ovarian carcinomas were stratified according to AEG-1 protein expression (Fig. 1E). Univariate analysis using the Kaplan-Meier method indicated an inverse correlation between AEG-1 expression and survival rate of patients with ovarian carcinoma $(\mathrm{P}=0.002)$. Multivariate analysis using Cox proportional hazard model indicated that AEG-1 expression $(\mathrm{P}=0.036)$ and FIGO staging $(\mathrm{P}=0.020)$ were independent prognostic factors for the overall survival of the patients with ovarian cancer (Table III).

Effects of AEG-1 on cell proliferation. To further investigate the biological role of AEG-1 expression on the progression of ovarian cancer, the impact of AEG-1 expression on ovarian cancer cell proliferation was evaluated in AEG-1 knockdown cells. The protein and mRNA expression levels of AEG-1 were decreased in the AEG-1-siRNA-transfected cells, as compared with the NC-transfected cells (Fig. 2A and B). Transfection with AEG-1-siRNA significantly decreased cell proliferation in the SKOV-3 cells at $96 \mathrm{~h}$, as compared with the control group ( $\mathrm{P}=0.001$, Fig. $2 \mathrm{C}$ ). To determine the mechanisms involved in the inhibition of proliferation, cell cycle distribution was analyzed using flow cytometry. Knockdown of 
Table III. Multivariate analysis of clinicopathological variables for the overall survival of the patients with ovarian cancer.

\begin{tabular}{lcr}
\hline Variable & Relative risk (95\% confidence interval) & P-value \\
\hline Age (years) & $1.026(0.980-1.074)$ & 0.272 \\
Histological type & $0.773(0.462-1.292)$ & 0.326 \\
Differentiation degree & $0.912(0.395-2.108)$ & 0.830 \\
Lymph node metastasis & $1.202(0.485-2.977)$ & 0.691 \\
Clinical stage & $2.268(1.141-4.508)$ & 0.020 \\
Astrocyte-elevated gene-1 & $3.037(1.067-8.670)$ & 0.036
\end{tabular}

A

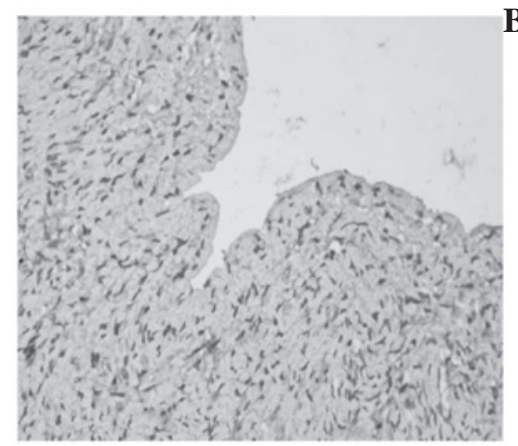

C

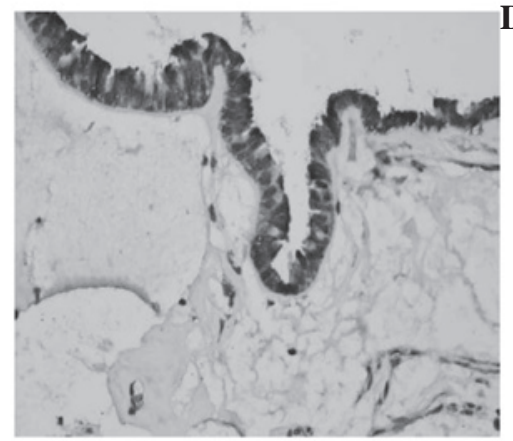

$\mathbf{E}$

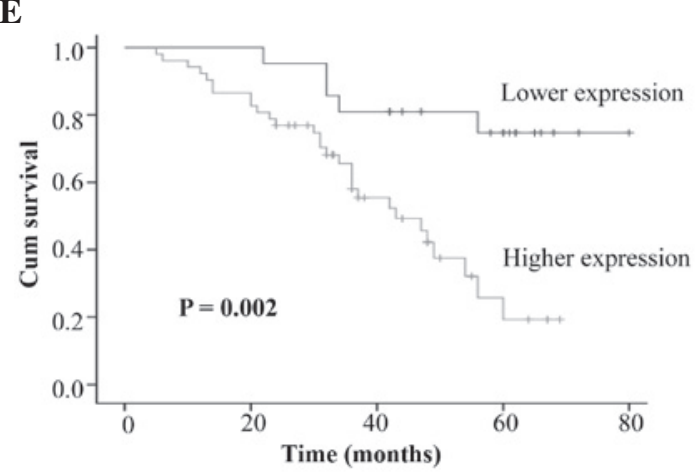

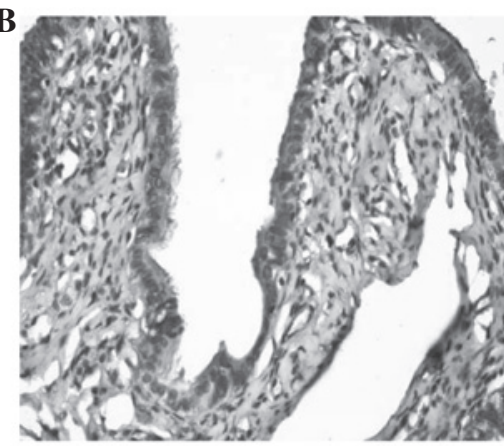

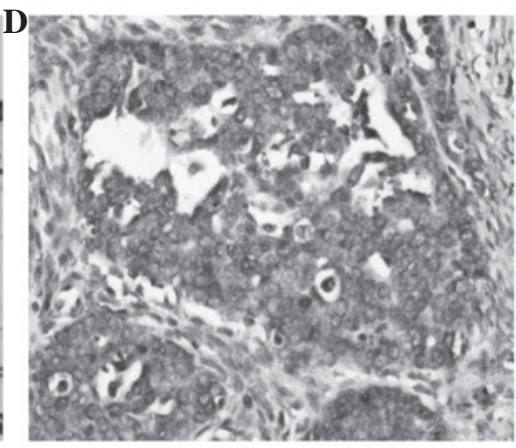

Figure 1. Immunohistochemical staining of astrocyte-elevated gene-1 (AEG-1) in ovarian tissues. (A) Little or no expression of AEG-1 was observed in the epithelial cells of normal ovarian tissue. (B) High expression levels of AEG-1 were detected in a cystadenoma, (C) ovarian borderline tumor and (D) ovarian carcinoma. (E) Kaplan-Meier analysis of the overall survival rate associated with AEG-1 expression. Overall survival curves of the 73 patients with ovarian carcinoma were grouped according to their AEG-1 expression status. $\mathrm{P}=0.002$.

AEG-1 expression resulted in an increased number of cells in the $\mathrm{G}_{0} / \mathrm{G}_{1}$ phase.

Furthermore, the impact of AEG-1 expression on ovarian cancer proliferation was also evaluated in the cells overexpressing AEG-1. Following transfection, western blot analysis was performed to analyze the protein expression levels of AEG-1. The protein expression levels of AEG-1 were increased in the AEG-1-transfected cells, as compared with the pcDNA3.1-transfected control cells (Fig. 3A). The cell proliferation assay indicated that AEG-1-transfected cells grew faster, as compared with those transfected with the vector control by day 3 after plating $(\mathrm{P}<0.001$, Fig. 3B). Furthermore, exogenous overexpression of AEG-1 reduced the population of cells within the $G_{0} / G_{1}$ phase of the cell cycle (Fig. 3C). 
A

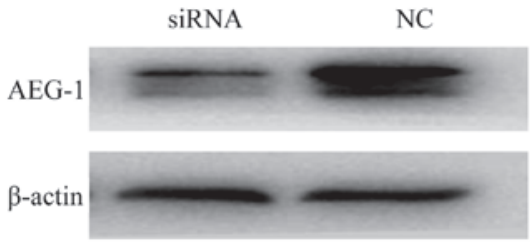

B

C
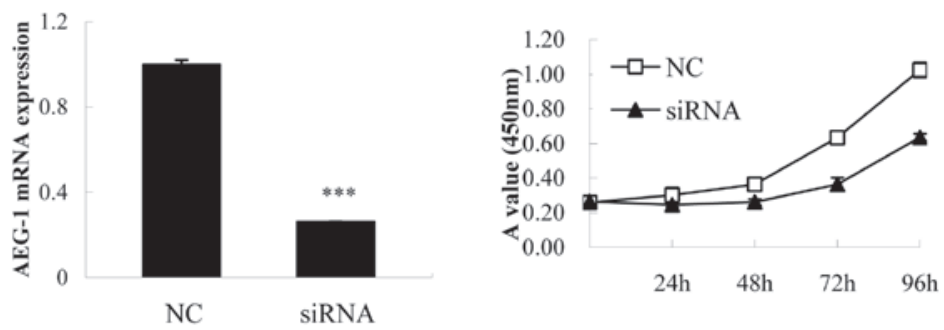

D
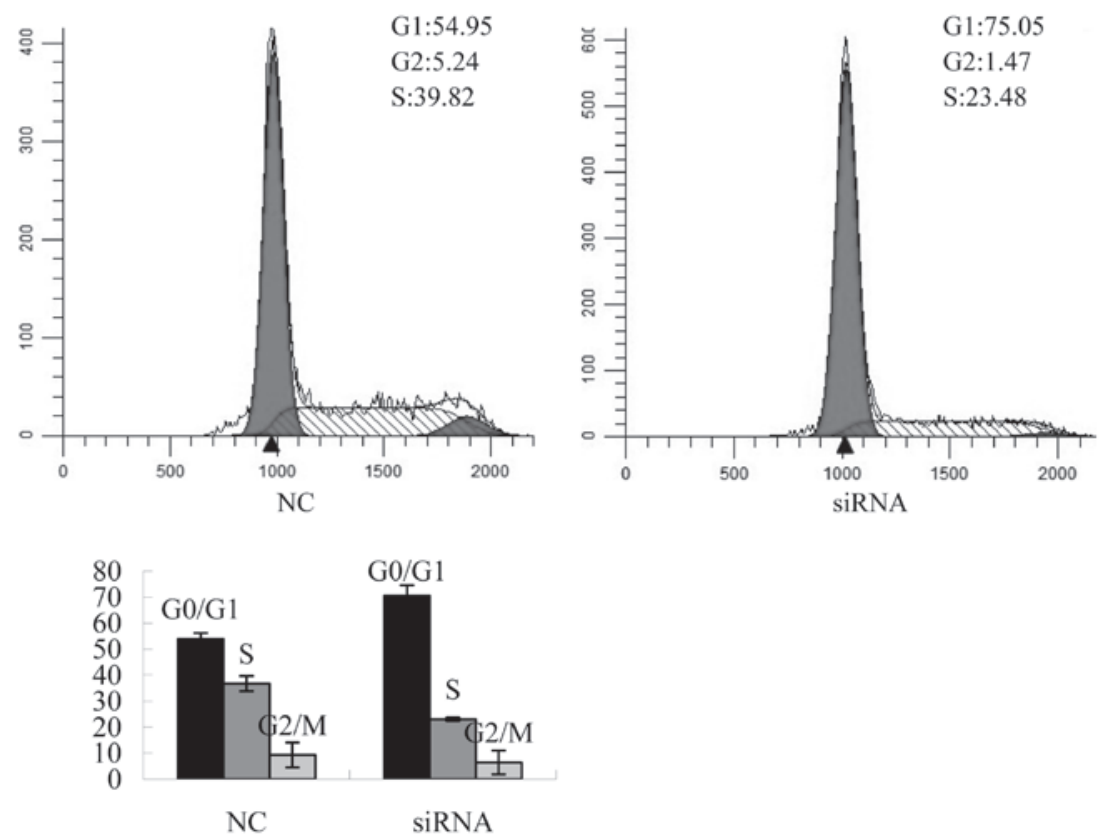

Figure 2. Downregulation of astrocyte elevated gene-1 (AEG-1), by small interfering (si)RNA in SKOV-3 human ovarian carcinoma cells, was detected by (A) western blot analysis and (B) reverse transcription-quantitative polymerase chain reaction (RT-qPCR). GAPDH was used as an internal control in RT-qPCR. (C) Silencing endogenous AEG-1 expression inhibited cell growth, as determined by Cell Counting kit-8 assay. (D) Knockdown of AEG-1 resulted in an increased number of cells in the $\mathrm{G}_{0} / \mathrm{G}_{1}$ phase. Data are presented as the mean \pm standard error of the mean of three independent experiments. ${ }^{* * * *} \mathrm{P}<0.001$ vs. the negative control (NC).

Effects of AEG-1 on cell adhesion. To explore the effects of AEG-1 on cell adhesion, an adhesion assay was performed using Matrigel ${ }^{\mathrm{TM}}$, which contains the majority of the components of the extracellular matrix. The adhesive capabilities of the AEG-1-siRNA-transfected cells were significantly reduced, as compared with the $\mathrm{NC}$-transfected cells $(\mathrm{P}=0.021$, Fig. 4A), whereas cell adhesion was significantly increased in the AEG-1-transfected cells, as compared with the vector control cells $(\mathrm{P}<0.001$, Fig. 4B).

Effects of AEG-1 on cell invasion. Transwell ${ }^{\circledR}$ assays were carried out, in order to determine the effects of AEG-1 on the invasive abilities of the ovarian cancer cells. In the AEG-1-siRNA-transfected cells, there was a $60.9 \%$ reduction in the number of invading cells, as compared with the NC-transfected cells $(\mathrm{P}<0.001$, Fig. $4 \mathrm{C})$, whereas the AEG-1-transfected cells exhibited a 2.1-fold increase in the number of invading cells, as compared with the pcDNA3.1-transfected cells $(\mathrm{P}<0.001$, Fig. 4D).

Downstream signaling activated by AEG-1.Signaling pathways activated by AEG-1 were analyzed by determining the protein expression levels of various forms of ERK1/2, FOXO3a, AKT, and GSK $3 \beta$ by western blot analysis. The protein expression levels of phosphorlyated FOXO3a and ERK1/2 were decreased in the AEG-1 knockdown cells, whereas the expression levels of phosphorylated ERK1/2 and phosphorylated FOXO3a in the AEG-1-overexpressing cells were increased, as compared with the vector control cells (Fig. 5). There were no significant changes in the expression levels of total FOXO3a and ERK1/2. In addition, AEG-1 deregulation did not affect the expression levels of phosphorylated AKT and phosphorylated GSK3 $\beta$. 
A

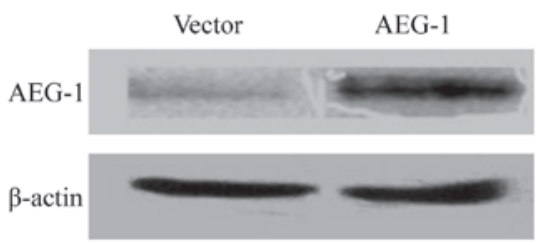

B

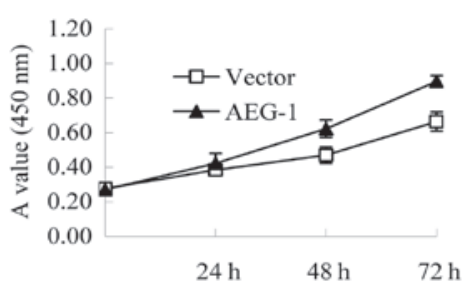

C
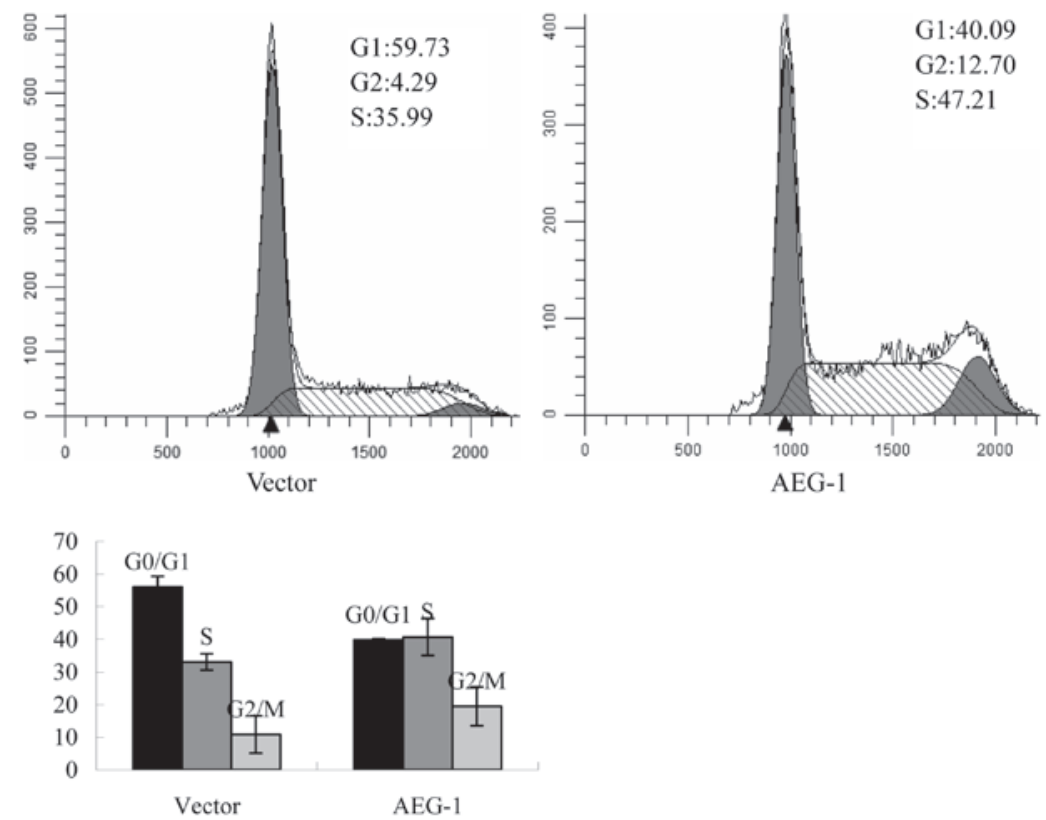

Figure 3. Upregulation of astrocyte elevated gene-1 (AEG-1) in SKOV3 human ovarian carcinoma cells (A) analyzed by western blotting. (B) Ectopic overexpression of AEG-1 stimulates cell proliferation, as determined by Cell Counting kit- 8 assays. (C) Exogenous overexpression of AEG-1 reduced the population of cells within the $\mathrm{G}_{0} / \mathrm{G}_{1}$ phase of the cell cycle. Data are presented as the mean \pm standard error of the mean of three independent experiments.

\section{Discussion}

The present study determined the expression levels of AEG-1 in 73 ovarian cancer specimens using IHC, and correlated the expression with the clinicopathological characteristics of the patients. AEG-1 was shown to be overexpressed in $71.2 \%$ of ovarian cancer specimens, whereas very little or no immunoreactivity was detected in the normal ovarian tissue. The protein expression levels of AEG-1 in the histological sections were closely correlated with the degree of differentiation, lymph node metastasis and clinical staging. These results suggest that upregulated expression of AEG-1 in ovarian cancer may facilitate the increased malignant phenotype of the tumor. In addition, patients with higher AEG-1 expression also had a shorter overall survival, as compared with the patients with lower expression. A multivariate analysis also indicated that AEG-1 may be an independent prognostic factor for the overall survival of patients with ovarian cancer. These results indicate that AEG-1 may represent a valuable biomarker for the prediction of ovarian cancer prognosis.
The results of the present study are concordant with the results of previous studies examining the role of AEG-1 in the progression of ovarian cancer $(15,24)$. In these previous studies, AEG-1 was shown to be overexpressed in metastatic tissues from patients with ovarian cancer. In addition, the overexpression of AEG-1 was correlated with peritoneal dissemination, lymph node metastasis, FIGO stage, histological grade, presence of residual tumor and tumor recurrence in ovarian cancer $(15,24)$. Meng et al (24), demonstrated that patients with high AEG-1 expression had significantly poorer overall survival, as compared with patients with low expression, which was similar to the findings of the present study. In addition, AEG-1 has been reported to be overexpressed in other cancer types, including liver cancer, breast cancer, esophageal squamous cell carcinoma, gastric cancer and non-small cell lung cancer $(10-12,25,26)$, in which overexpression of AEG-1 was often observed in more aggressive tumor subgroups, and is therefore considered to have diagnostic value.

The present study demonstrated that an increasing frequency of overexpression of AEG-1 was observed from 
A

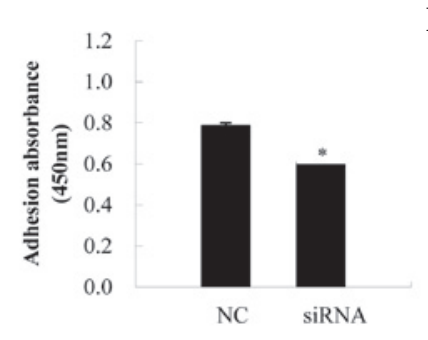

C

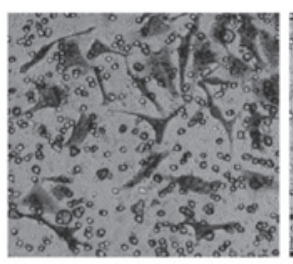

$\mathrm{NC}$

D

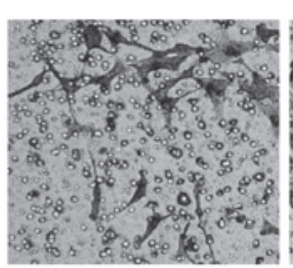

Vector

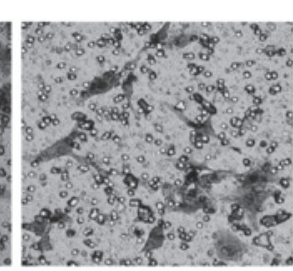

siRNA

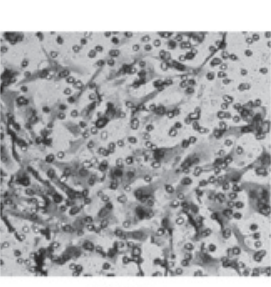

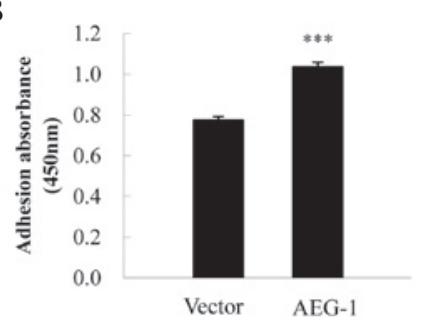
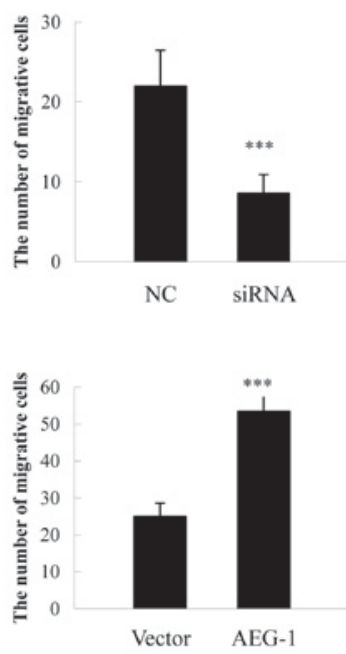

Figure 4. Effects of AEG-1 on SKOV-3 human ovarian carcinoma cells in vitro. (A and B) AEG-1 modulates the adhesive and (C and D) invasive abilities of SKOV-3 cells. ${ }^{*} \mathrm{P}<0.05 ;{ }^{* * * *} \mathrm{P}<0.001$. AEG-1, astrocyte-elevated gene-1; NC, negative control; siRNA, small interfering RNA.

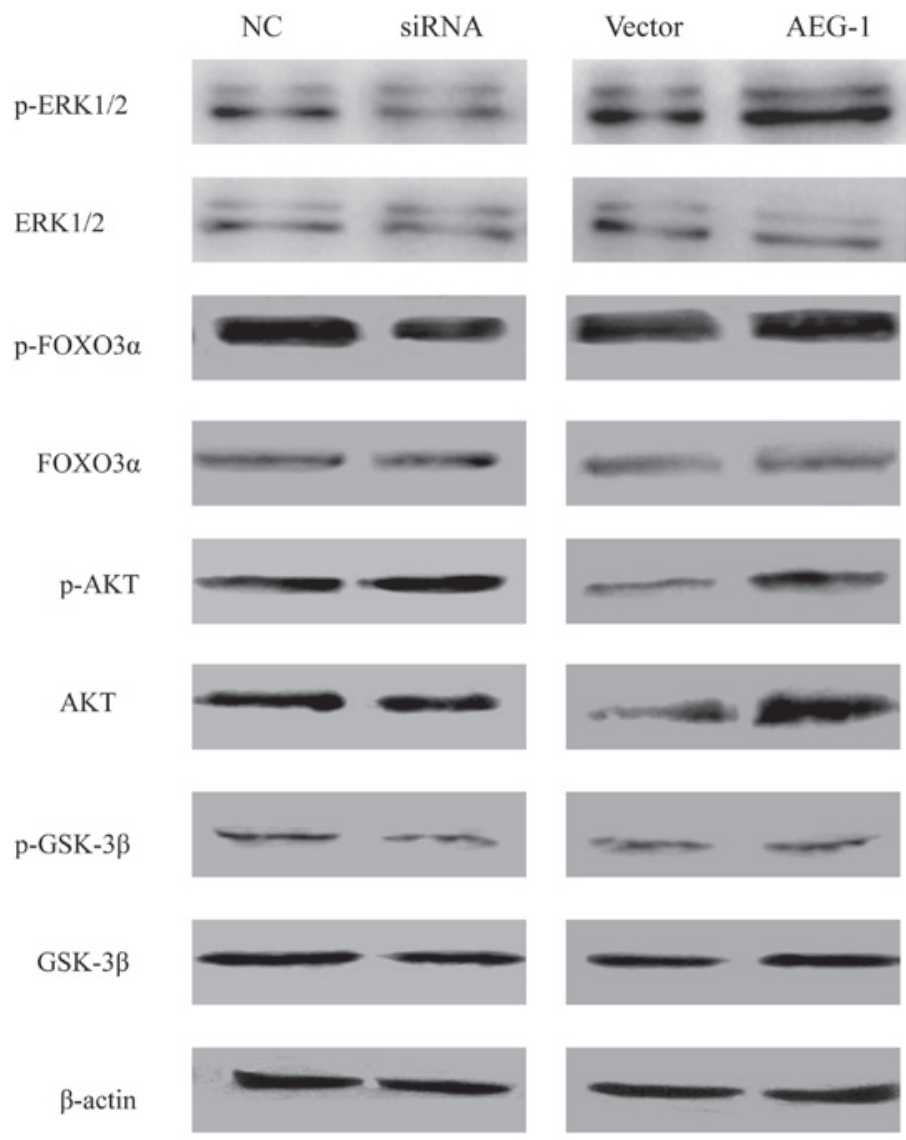

Figure 5. Expression analysis of various forms of ERK1/2, FOXO3 $\alpha$, AKT, and GSK3 $\beta$ proteins by western blotting. Protein expression levels were normalized to $\beta$-actin expression levels in each sample. NC, negative control; siRNA, small interfering RNA; ERK, extracellular signal-regulated kinase; FOXO3, forkhead box O3; GSK, glycogen synthase kinase; p-, phosphorylated-; AEG-1, astrocyte-elevated gene-1. 
benign (cystadenoma) to borderline tumors, and to malignant carcinomas. This is the first time this has been reported in ovarian cancer, to the best of our knowledge. This finding has however been reported in other cancer types, such as colorectal and breast cancer $(13,27)$. These data suggest that AEG-1 may have an important role in the tumorigenic process of human cancers, including ovarian cancer.

Recently, numerous studies have shown that AEG-1 is associated with biological processes including cancer cell proliferation, apoptosis, migration, and metastasis (16-19). However, thus far, there have been few reports regarding the regulation and function of AEG-1 in ovarian cancer. Therefore, the present study investigated the gain or loss of AEG-1 function, through exogenous overexpression or AEG-1 knockdown by siRNA, in ovarian cancer cells. Exogenous overexpression of AEG-1 in ovarian cancer cells significantly enhanced cell proliferation and reduced the $\mathrm{G}_{0} / \mathrm{G}_{1}$ cell population. Conversely, silencing AEG-1 expression resulted in a clear inhibition of cell growth and induced a cell cycle arrest at the $G_{0} / G_{1}$ phase. In addition, upregulation of AEG-1 in ovarian cancer cells resulted in improved adhesive and invasive capabilities, whereas, downregulation of AEG-1 reduced adhesion and inhibited invasion of the cells. These data not only support the finding that AEG-1 overexpression is associated with poor prognosis in ovarian cancer, but also implicate an association between the function of AEG-1 and the pathogenesis of ovarian cancer. These results may lead to the development of a novel therapeutic strategy against ovarian cancer. Concordant with the findings of the present study, these observations have also been reported in hepatocellular carcinoma, neuroblastoma, malignant glioma cells and colorectal carcinoma (12,28-31).

In recent years, the function of AEG-1 has been intensively investigated; however, the molecular mechanisms underlying its oncogenic role remain unclear. There has been abundant evidence demonstrating that numerous major cellular signaling pathways, including PI3K/AKT, ERK1/2, and p38MAPK, may have important roles in the ability of AEG-1 to execute the identified biological functions in various cancers. Through activation of the PI3K/AKT signaling pathway, AEG-1 overexpression could block the serum starvation-induced cell death through phosphorylation of GSK3 $\beta$, Bcl-2-associated death promoter and mouse double minute 2 homolog, and reduction of p53 and p21/mda- 6 expression (32). In esophageal squamous cancer cells, AEG-1 was shown to decrease the expression of p27Kip1 and upregulate the expression of cyclin D1, through the AKT/FOXO3a pathway (11). Activation of AKT by AEG-1 in breast cancer cells resulted in a downregulation of the transcriptional activity of FOXO1, and reduction of two key cell-cycle inhibitors: p27Kip1 and p21Cip1 (33). In hepatocellular carcinoma, AEG-1 has been shown to increase phosphorylation of MAPK molecules, including ERK1/2 and p38MAPK, which subsequently activates Wnt-mediated signaling and consequently leads to increased tumor angiogenesis (12). The present study demonstrated that AEG-1 knockdown could decrease the phosphorylation levels of ERK1/2 and FOXO3a, without affecting the expression levels of phosphorylated AKT and GSK3 $\beta$. Therefore, it may be speculated that the effects of AEG-1 on the proliferation and tumorigenicity of ovarian cancer cells may be associated with the activity of ERK1/2 and FOXO3a. However, although numerous signaling pathways involved in mediating the molecular functions of AEG-1 have been elucidated, it remains unclear what direct interactions occur between AEG-1 and other proteins, and how they contribute to the downstream effects of AEG-1. A future aim may be to identify the interacting partners of AEG-1, using numerous methods.

In conclusion, the present study identified that the expression levels of AEG-1 were highly increased in ovarian cancer tissue. Furthermore, AEG-1 protein expression was significantly correlated with survival and malignant metastasis of ovarian cancer. The present study further elucidates the molecular mechanisms underlying invasion and metastasis of ovarian cancer. These data suggest that AEG-1 may represent a valuable biomarker for the prediction of ovarian cancer prognosis, and an attractive molecular target for novel anticancer therapeutic agents.

\section{References}

1. Jemal A, Bray F, Center MM, Ferlay J, Ward E and Forman D: Global cancer statistics. CA Cancer J Clin 61: 69-90, 2011.

2. Poveda A: Ovarian cancer: is the news good enough? Int J Gynecol Cancer 15: 298-306, 2005.

3. Seidman JD, Horkayne-Szakaly I, Haiba M, Boice CR, Kurman RJ and Ronnett BM: The histologic type and stage distribution of ovarian carcinomas of surface epithelial origin. Int J Gynecol Pathol 23: 41-44, 2004.

4. Cheng W, Liu J, Yoshida H, Rosen D and Naora H: Lineage infidelity of epithelial ovarian cancers is controlled by HOX genes that specify regional identity in the reproductive tract. Nat Med 11: 531-537, 2005.

5. Zhou B, Yang L, Wang L, et al: The association of tea consumption with ovarian cancer risk: A meta-analysis. Am J Obstet Gynecol 197: 594. e1-e6, 2007.

6. Siegel R, Naishadham D and Jemal A: Cancer statistics 2012. CA Cancer J Clin 62: 10-29, 2012.

7. Saad AF, Hu W and Sood AK: Microenvironment and pathogenesis of epithelial ovarian cancer. Horm Cancer 1: 277-290, 2010.

8. Su ZZ, Kang DC, Chen Y, et al: Identification and cloning of human astrocyte genes displaying elevated expression after infection with HIV-1 or exposure to HIV-1 envelope glycoprotein by rapid subtraction hybridization, RaSH. Oncogene 21: 3592-3602, 2002.

9. Kang DC, Su ZZ, Sarkar D, Emdad L, Volsky DJ and Fisher PB: Cloning and characterization of HIV-1-inducible astrocyte elevated gene-1, AEG-1. Gene 353: 8-15, 2005.

10. Li J, Zhang N, Song LB, et al: Astrocyte elevated gene-1 is a novel prognostic marker for breast cancer progression and overall patient survival. Clin Cancer Res 14: 3319-3326, 2008.

11. Yu C, Chen K, Zheng $\mathrm{H}$ et al: Overexpression of astrocyte elevated gene-1 (AEG-1) is associated with esophageal squamous cell carcinoma (ESCC) progression and pathogenesis. Carcinogenesis 30: 894-901, 2009.

12. Yoo BK, Emdad L, Su ZZ, et al: Astrocyte elevated gene-1 regulates hepatocellular carcinoma development and progression. J Clin Invest 119: 465-477, 2009.

13. Song H, Li C, Li R and Geng J: Prognostic significance of AEG-1 expression in colorectal carcinoma. Int J Colorectal Dis 25: 1201-1209, 2010

14. Kikuno N, Shiina H, Urakami S, et al: Knockdown of astrocyte-elevated gene-1 inhibits prostate cancer progression through upregulation of FOXO3a activity. Oncogene; 26: 7647-7655, 2007.

15. Li C,Liu J,Lu R, et al: AEG -1 overexpression: a novel indicator for peritoneal dissemination and lymph node metastasis in epithelial ovarian cancers. Int J Gynecol Cancer 21: 602-608, 2011.

16. Hu G, Chong RA, Yang Q, et al: MTDH activation by $8 \mathrm{q} 22$ genomic gain promotes chemoresistance and metastasis of poor-prognosis breast cancer. Cancer Cell 15: 9-20, 2009.

17. Yoo BK, Chen D, Su ZZ, et al: Molecular mechanism of chemoresistance by astrocyte elevated gene-1. Cancer Res 70: 3249-3258, 2010 . 
18. Emdad L, Lee SG, Su ZZ, et al: Astrocyte elevated gene-1 (AEG-1) functions as an oncogene and regulates angiogenesis. Proc Natl Acad Sci USA 106: 21300-21305, 2009.

19. Emdad L, Sarkar D, Su ZZ, et al: Activation of the nuclear factor kappaB pathway by astrocyte elevated gene-1: implications for tumor progression and metastasis. Cancer Res 66: 1509-1516, 2006.

20. Lee SG, Su ZZ, Emdad L, Sarkar D and Fisher PB: Astrocyte elevated gene-1 (AEG-1) is a target gene of oncogenic Ha-ras requiring phosphatidylinositol 3-kinase and c-Myc. Proc Natl Acad Sci USA 103: 17390-17395, 2006.

21. Scully RE (ed): Histological Typing of Ovarian Tumors. World Health Organization. International Histological Classification of Tumours. Springer, Berlin, Germany, 1999.

22. Berg D, Malinowsky K, Reischauer B, Wolff C and Becker KF: Use of formalin-fixed and paraffin-embedded tissues for diagnosis and therapy in routine clinical settings. Methods Mol Biol 785: 109-122, 2011.

23. Schefe JH, Lehmann KE, Buschmann IR, Unger T and Funke-Kaiser H: Quantitative real-time RT-PCR data analysis: current concepts and the novel 'gene expression's CT difference' formula. J Mol Med (Berl) 84: 901-910, 2006.

24. Meng F, Luo C, Ma L, Hu Y and Lou G: Clinical significance of astrocyte elevated gene-1 expression in human epithelial ovarian carcinoma. Int J Gynecol Pathol 30: 145-150, 2011.

25. Jian-bo X, Hui W, Yu-long H, et al: Astrocyte-elevated gene-1 overexpression is associated with poor prognosis in gastric cancer. Med Oncol 28: 455-462, 2011.
26. Ke ZF, Mao X, Zeng C, He S, Li S and Wang LT: AEG-1 expression characteristics in human non-small cell lung cancer and its relationship with apoptosis. Med Oncol 30: 383, 2013.

27. Su P, Zhang Q and Yang Q: Immunohistochemical analysis of Metadherin in proliferative and cancerous breast tissue. Diagn Pathol 5: 38, 2010.

28. Lee SG, Jeon HY, Su ZZ, et al: Astrocyte elevated gene-1 contributes to the pathogenesis of neuroblastoma. Oncogene 28: 2476-2484, 2009.

29. Emdad L, Sarkar D, Lee SG et al: Astrocyte elevated gene-1: A novel target for human glioma therapy. Mol Cancer Ther 9: 79-88, 2010.

30. Liu L, Wu J, Ying Z, et al: Astrocyte elevated gene-1 upregulates matrix metalloproteinase-9 and induces human glioma invasion. Cancer Res 70: 3750-3759, 2010.

31. Zhang F, Yang Q, Meng F, et al: Astrocyte elevated gene-1 interacts with $\beta$-catenin and increases migration and invasion of colorectal carcinoma. Mol Carcinog 52: 603-610, 2013.

32. Lee SG, Su ZZ, Emdad L, Sarkar D, Franke TF and Fisher PB: Astrocyte elevated gene-1 activates cell survival pathways through PI3K-Akt signaling. Oncogene 27: 1114-1121, 2008.

33. Li J, Yang L, Song L, et al: Astrocyte elevated gene-1 is a proliferation promoter in breast cancer via suppressing transcriptional factor FOXO1. Oncogene 28: 3188-3196, 2009. 